INFECTION CONTROL AND HOSPITAL EPIDEMIOLOGY MARCH 2013, VOL. 34 , No. 3

\title{
Modified World Health Organization Hand Rub Formulations Comply with European Efficacy Requirements for Preoperative Surgical Hand Preparations
}

\author{
Miranda Suchomel, PhD; ${ }^{1}$ Michael Kundi, $\mathrm{PhD} ;^{2}$ Didier Pittet, MD; ${ }^{3,4,5}$ Manfred L. Rotter, MD
}

\begin{abstract}
васKGround. The World Health Organization (WHO) has published "Guidelines on Hand Hygiene in Health Care" recommending 2 hand rub formulations based on $80 \% \mathrm{vol} / \mathrm{vol}$ ethanol or $75 \% \mathrm{vol} / \mathrm{vol}$ isopropanol for local production in healthcare settings where commercial products are not available or are too expensive. Previous investigations have shown that neither formulation meets the efficacy requirements of European norm (EN) 12791, which is the most stringent available norm for surgical hand rub preparations. Even when modified with approximately $5 \%$ higher alcohol content, the formulations proved to be inferior to the reference of the norm when measured after 3 hours.
\end{abstract}

овJECTIVE. Because the high glycerol content of the formulations was suspected to negatively influence their efficacy, additional investigations were performed with varying glycerol content.

METHODS. Modified formulations with higher alcohol concentration (mass instead of volume percentage) and lower glycerol concentration $(0.725 \%$ instead of $1.45 \%)$ or without the addition of glycerol were evaluated for their conformity with the efficacy requirements of EN 12791, which demands noninferiority in comparison with a reference hand antisepsis procedure immediately and 3 hours after treatment on volunteers' hands.

DESIGN. Randomized Latin-square design.

Setting. Microbiology laboratory of the Medical University of Vienna, Vienna, Austria.

PARTICIPANTs. Twenty-five healthy volunteers.

RESULTS. Reducing the concentration of glycerol or omitting it completely rendered both WHO formulations noninferior to the reference, both immediately and 3 hours after surgical hand antisepsis.

concLusions. Both WHO-recommended formulations meet the efficacy requirements of EN 12791 by increasing their alcohol concentrations by $5 \%$, prolonging their application to 5 minutes and reducing the glycerol concentration to $0.725 \%$.

Infect Control Hosp Epidemiol 2013;34(3):245-250

In 2009, the World Health Organization (WHO) published "Guidelines on Hand Hygiene in Health Care," which recommended the use of alcohol-based hand rubs for both hygienic and preoperative hand treatment. For local production in healthcare settings where commercial products are not available or too expensive, the guideline recommended 2 different hand rub formulations based on either ethanol $80 \%$ $\mathrm{vol} / \mathrm{vol}$ (WHO I) or isopropanol $75 \% \mathrm{vol} / \mathrm{vol}$ (WHO II) as active agents. Additionally, low concentrations of hydrogen peroxide $(0.125 \% \mathrm{vol} / \mathrm{vol})$ are incorporated in the formulations to destroy potentially contaminating bacterial spores during storage. Furthermore, glycerol $(1.45 \% \mathrm{vol} / \mathrm{vol})$ is added as an emollient to improve dermal acceptability.

Previous investigations have shown that neither formulation meets the efficacy requirements of the European norm (EN) $12791,{ }^{2}$ which is the most stringent in vivo laboratory assay for testing products with respect to their bactericidal efficacy in surgical hand treatment. ${ }^{3,4}$ This norm requires that the reduction of skin bacteria from the hands of volunteers caused by the product shall not be inferior to that achieved with a standard reference procedure, rubbing n-propanol $60 \%(\mathrm{vol} / \mathrm{vol})$ onto the hands of the same subjects for 3

Affiliations: 1. Institute of Hygiene and Applied Immunology, Medical University of Vienna, Vienna, Austria; 2. Center for Public Health, Medical University of Vienna, Vienna, Austria; 3. First Global Patient Safety Challenge, World Health Organization Patient Safety, World Health Organization, Geneva, Switzerland; 4. Infection Control Programme, University of Geneva Hospitals and Faculty of Medicine, Geneva, Switzerland; 5. World Health Organization Collaborating Centre on Patient Safety, University of Geneva Hospitals and Faculty of Medicine, Geneva, Switzerland.

Received August 6, 2012; accepted October 28, 2012; electronically published January 18, 2013.

(C) 2013 by The Society for Healthcare Epidemiology of America. All rights reserved. 0899-823X/2013/3403-0004\$15.00. DOI: 10.1086/669528 
minutes. The 2009 WHO guideline itself reported that WHO I did not exceed the efficacy of the norm in 2 test laboratories and that WHO II did not exceed the efficacy of the norm in 1 of 2 test laboratories. ${ }^{1}$ To improve the bactericidal efficacy of the formulations, we modified both formulations by increasing their alcohol concentrations by approximately $5 \%$, changing their volume percentage into weight percentage, and prolonging the duration of application from 3 to 5 minutes, which is the longest duration of application allowed by the norm. It is of note that a mass concentration of $80 \%$ ethanol equals a volume concentration of approximately $85 \%$ ethanol, whereas a mass concentration of $75 \%$ isopropanol is equivalent to a volume concentration of approximately $80 \%$ isopropanol. These modifications were earlier shown to render the immediate effect of both formulations noninferior to the reference of EN 12791 on an ungloved hand tested immediately after antisepsis. ${ }^{4}$ Surprisingly, this improvement was not observed when the formulations were tested on a gloved hand and measured 3 hours after treatment; with respect to the so-called 3-hour effect, the efficacy of the formulations still proved inferior to that of the corresponding reference value. ${ }^{4}$ Because the high glycerol concentration $(1.45 \% \mathrm{vol} /$ vol) of the formulations was suspected to exert a negative influence on their 3-hour efficacy, we performed additional studies in which the glycerol was reduced by half or omitted entirely.

\section{METHODS}

The following formulations were used: EtOH G+, consisting of ethanol (pro analysi; Merck) $80 \%$ (wt/wt), hydrogen peroxide (pro analysi; Merck) $0.125 \%$ (vol/vol), and glycerol (pro analysi; Merck) $0.725 \%$ ( $\mathrm{vol} / \mathrm{vol}$ ); EtOH G-, consisting of ethanol $80 \%$ (wt/wt), hydrogen peroxide $0.125 \%$ ( $\mathrm{vol} / \mathrm{vol}$ ), NO glycerol; Iso $\mathrm{G}+$, consisting of isopropanol (pro analysi; Merck) $75 \%(\mathrm{wt} / \mathrm{wt})$, hydrogen peroxide $0.125 \%(\mathrm{vol} / \mathrm{vol})$, and glycerol $0.725 \%(\mathrm{vol} / \mathrm{vol})$; and Iso $\mathrm{G}-$, consisting of isopropanol $75 \%(\mathrm{wt} / \mathrm{wt}$ ), hydrogen peroxide $0.125 \%$ ( $\mathrm{vol} /$ vol), and NO glycerol. N-propanol (pro analysi; Merck) $60 \%$ ( $\mathrm{vol} / \mathrm{vol}$ ) without additions served as the reference antiseptic as specified in EN $12791 .^{2}$

Twenty-five study subjects, all of whom were employees of the Institute for Hygiene and Applied Immunology, Medical University of Vienna (Vienna, Austria), participated as volunteers in this study. All had given their written informed consent. The study protocol was approved by the institutional ethics committee of the Medical University of Vienna. Exclusion criteria were as follows: age less than 18 years, pregnancy, and skin breaks on hands (such as cuts, abrasions, and skin disorders). Nails were short and clean, and the study subjects agreed not to use any antibacterial chemicals or antibacterial soap during the trial, starting from 1 week before testing.

Culture media were those described in EN 12791. Sampling and dilution fluids were Tryptic Soy Broth (Caso broth;
Merck). Counting plates were Tryptic Soy Agar (TSA; Caso agar; Merck). Neutralizing agents were not necessary for any of the tested formulations, because even dilution with pure broth lacking any supplement has been shown in many previous validation tests to neutralize any antimicrobial effect.

The bactericidal efficacies of the formulations were compared in 5 individual test runs with the standardized reference surgical hand treatment of EN 12791. A Latin-square design was used with 5 groups, each of 5 randomly allocated subjects, and as many experimental runs as there were formulations, including the reference. In every run, all hand treatment procedures were tested concurrently. At the end of the fifth test run, every subject had used each formulation once. The test runs were separated by 1 week to allow regrowth of the normal skin flora.

As with the evaluations previously published by Kampf et $\mathrm{al}^{3}$ and by us, ${ }^{4}$ the test method was that described in the forthcoming amendment of EN $12791,{ }^{2}$ which differs from the current version of EN 12791 mainly in the statistical processing of the results by employing a noninferiority test model, rather than the presently used comparative test model. To remove transient bacterial flora and any foreign material, a 1-minute preparatory hand wash was performed with nonmedicated soap and water. Hands were rinsed under running tap water and dried with paper towels. For the assessment of the pretreatment values, samples were obtained by rubbing and kneading the fingertips, including the thumbs, of both hands for 1 minute at the base of a petri dish $(9 \mathrm{~cm}$ in diameter) containing $10 \mathrm{~mL}$ of sampling fluid. A separate dish was used for each hand. Subsequently, hand antisepsis was performed according to the standardized hand rub procedure of EN 12791. This consisted of applying and rubbing as many 3-mL portions of the study formulations (EtOH G + , EtOH G-, Iso $\mathrm{G}+$, Iso $\mathrm{G}-$ ) onto both hands up to the wrists as were necessary to keep the hands wet for a total of 5 minutes, or 3 minutes for the reference procedure of EN 12791. For the assessment of posttreatment values, the fingertips of a randomly selected hand were sampled immediately after treatment as described above for the pretreatment values. The second hand was gloved and sampled after 3 hours for the assessment of 3-hour efficacy. Quantitative surface cultures from all sampling fluids and their decimal dilutions were performed on TSA. Counting plates were incubated at a mean temperature $\left( \pm\right.$ standard deviation) of $36^{\circ} \pm 1^{\circ} \mathrm{C}$ for a total of 48 hours, and colony-forming units were counted by means of an electronic colony counter (Fisher colony counter, Model 480; Artek Systems). Allowing for the dilution of the sampling fluids, the number of colony-forming units per milliliter was calculated.

For statistical evaluation, all pre- and posttreatment colony counts per milliliter of sampling fluid were expressed as decadic logarithms $\left(\log _{10}\right)$. From the intra-individual differences between $\log _{10}$ pretreatment minus $\log _{10}$ posttreatment values, individual $\log _{10}$ reduction factors $\left(\log _{10}\right.$ RFs) were calculated separately for both the immediate and the 3-hour effects. 
Pretreatment values of the study formulations and the reference were tested for significant differences by repeatedmeasures analysis of variance (ANOVA) at an agreed level of significance of $P=.05$. Subsequently, the intraindividual differences between the $\log _{10}$ RFs from each test formulation and the appropriate values of the reference were computed separately for both immediate and 3-hour effects and tested for significance by a nonparametric noninferiority test according to Hodges-Lehmann. ${ }^{5}$ Inferiority of a study formulation was rejected and noninferiority assumed if the HodgesLehmann upper $97.5 \%$ confidence limits for the differences in $\log _{10}$ bacterial reductions between study formulations and reference treatment were smaller than the agreed inferiority margin of $0.75 \log _{100^{5}}$

In addition, to evaluate differences between the study formulations and also between their various components, such as factor EtOH versus Iso or $\mathrm{G}+$ versus $\mathrm{G}-$, ANOVAs with repeated measures were performed with the differences $(\Delta)$ of the respective $\log _{10} R F$ component of interest minus the $\log _{10} R F$ of the appropriate reference values. Additionally, tests for linear contrasts were performed with these differences, such as comparison of glycerol within the 2 alcohols, EtOH and Iso, or of the alcohols within the 2 glycerol concentrations ( $\mathrm{G}+$ and $\mathrm{G}-$ ). A significance level of $P=.05$ has been agreed upon. The tests were done separately for immediate and 3-hour effects. Furthermore, a Wilcoxon matched-pairs, signed-ranks test was used to test for a suspected sustained effect at $P=.01$ (1-sided) if, as was concluded from a higher mean $\log _{10}$ reduction, a study formulation was suspected to be more efficacious than the reference antisepsis procedure 3 hours after antisepsis.

\section{RESULTS}

No significant differences were seen between the means of the $\log _{10}$ pretreatment bacterial counts of both the tests for immediate and 3-hour efficacy $(P=.93$ and $P=.17$, respectively, by repeated-measures ANOVA; Tables 1 and 2). Hence, baseline for each formulation can be considered to be equivalent. The mean $\log _{10}$ bacterial reductions immediately and 3 hours after surgical hand treatment with the modified formulations and with the reference are shown in Tables 1 and 2. Although the statistical tests for noninferiority have been performed on the mean intra-individual differences between each study formulation and the appropriate reference, the results of both the mean $\log _{10}$ RFs of the study formulations and the mean $\log _{10}$ differences $(\Delta)$ from the appropriate reference values are shown together to give a clear synopsis of the test results and the results of the statistical analysis.

At both measurements, immediately and 3 hours after hand antisepsis, both variations of the ethanol-based formulations, EtOH G+ with and EtOH G - without glycerol, proved noninferior to the reference. At 3 hours, the mean $\log _{10}$ bacterial reduction of the formulation without glycerol $\left(\mathrm{EtOH} \mathrm{G}^{-}\right)$ was even significantly $(P<.01$ by Wilcoxon matched-pairs signed-ranks test) larger by $0.48 \log _{10}$ than that of the reference treatment, which suggests sustained efficacy.

The above overall test result was also observed with both variations of the isopropanol-based formulations, Iso G+ with glycerol and Iso $\mathrm{G}$ - without glycerol. Therefore, all modified test formulations met the efficacy requirements of EN 12791 for testing surgical hand preparation with the prolonged duration of application of 5 minutes.

With regard to the immediate effects (Table 1), formulations containing isopropanol (Iso) with or without glycerol were on the whole significantly ( $P=.01$ by ANOVA) more efficacious than the formulations with ethanol. However, when analyzing these results by tests for linear contrasts, this was true only for the formulations without glycerol, EtOH $\mathrm{G}-$ versus Iso $\mathrm{G}-(P=.009)$, whereas those with glycerol, EtOH $\mathrm{G}+$ versus Iso $\mathrm{G}+$, showed no significant differences $(P=.776)$. There was a tendency towards a stronger efficacy of formulations without glycerol ( $P=.074$ by ANOVA), but this difference was significant $(P=.015)$ only within the results from isopropanol-containing formulations, the latter causing an even stronger reduction than the reference.

On the whole, ANOVAs with repeated measures on the 3 hour effects (Table 2) of the modified formulations revealed a virtually identical performance of both alcohols. The difference between the formulations that contained glycerol and those that did not, however, was significant $(P=.005)$. At a closer look, these differences were nearly of the same magnitude for both alcohols but became significant $(P=.014)$ only between the isopropanol-based formulations, Iso $G+$ and Iso $\mathrm{G}-$, although the ethanol-based formulations EtOH $\mathrm{G}+$ and $\mathrm{EtOH} \mathrm{G}$ - also showed a tendency in this direction $(P=.08)$. It is noticeable that the 3-hour effects of both formulations lacking glycerol proved significantly $(P=.01)$ stronger than that of the reference.

\section{DISCUSSION}

As shown previously by Kampf et $\mathrm{al}^{3}$ and ourselves, ${ }^{4}$ both of the original WHO-recommended formulations containing EtOH or Iso do not meet the efficacy requirements of the forthcoming amendment of EN 12791 for surgical hand preparations. Even prolongation of the application to 5 minutes, which is the longest duration of application allowed by EN $12791,{ }^{2}$ did not result in a favorable outcome for these formulations. ${ }^{3,4}$ From the results of several of our earlier studies, we know that the bactericidal efficacy of alcohol-based hand rubs varies, not only with the type of alcohol and contact time, but also with the alcohol concentration. ${ }^{6-9}$

Recently, we have shown that pure ethanol met the efficacy requirement of EN 12791 at a concentration of $85 \%$ (vol/ vol), whereas it failed to meet that requirement at $75 \%$ (vol/ vol). ${ }^{9}$ In another study by Kampf et al, ${ }^{10}$ a hand rub based on $80 \% \mathrm{wt} / \mathrm{wt}$ ethanol was also found to be as effective as the reference of EN 12791. As with ethanol, the isopropanol 
TA B LE 1. Comparison of the Immediate Effects of a 5-Minute Application of Modified World Health Organization Antiseptic Hand Rub Formulations with 3-Minute Application of Reference Surgical Hand Antiseptic Treatment according to European Norm 12791

\begin{tabular}{|c|c|c|c|c|c|}
\hline \multirow[b]{2}{*}{ Formulation } & \multirow[b]{2}{*}{$\begin{array}{l}\text { Glycerol } \\
\text { added }\end{array}$} & \multicolumn{3}{|c|}{$\begin{array}{c}\text { Skin bacteria, } c f u / m L \\
(n=25)\end{array}$} & \multirow[b]{2}{*}{$\begin{array}{l}\text { Hodges-Lehmann upper } \\
97.5 \% \text { confidence limit }\end{array}$} \\
\hline & & $\begin{array}{c}\text { Mean } \log _{10} \\
\text { prevalue }( \pm S D)\end{array}$ & $\begin{array}{l}\text { Mean } \log _{10} \\
\text { reduction }( \pm S D)\end{array}$ & $\begin{array}{c}\text { Mean } \log _{10} \\
\text { difference }( \pm S D) \\
\Delta, \text { to reference }\end{array}$ & \\
\hline $\mathrm{EtOH} \mathrm{G+}$ & Yes & $4.60 \pm 0.59$ & $2.35 \pm 1.21$ & $0.06 \pm 0.76$ & 0.39 n.i. \\
\hline EtOH G- & No & $4.58 \pm 0.55$ & $2.37 \pm 0.94$ & $0.04 \pm 1.20$ & 0.49 n.i. \\
\hline Iso $\mathrm{G}+$ & Yes & $4.62 \pm 0.57$ & $2.41 \pm 1.01$ & $0.00 \pm 0.99$ & 0.38 n.i. \\
\hline Iso G- & No & $4.56 \pm 0.63$ & $2.95 \pm 1.08$ & $-0.53 \pm 1.04$ & -0.08 n.i. \\
\hline Reference & No & $4.54 \pm 0.69$ & $2.41 \pm 1.28$ & $\ldots$ & $\ldots$ \\
\hline
\end{tabular}

NOTE. cfu, colony-forming units; EtOH, ethanol $80 \%$ (wt/wt) plus hydrogen peroxide $0.125 \%$ (vol/vol); G+, with glycerol $0.725 \%$ (vol/vol); G-, without glycerol; Iso, isopropanol $75 \%$ (wt/wt) plus hydrogen peroxide $0.125 \%$ (vol/vol); n.i., noninferior versus reference; reference, n-propanol 60\% (vol/vol); SD, standard deviation.

a With an agreed inferiority margin of $0.75 \log _{10}$.

concentration $(75 \% \mathrm{vol} / \mathrm{vol})$ seemed to be too low in the original WHO II-recommended formulation. Rotter et $\mathrm{al}^{11}$ have shown that a $70 \%(\mathrm{vol} / \mathrm{vol})$ isopropanol-based hand rub was not as effective as the reference alcohol in the EN. In another study, pure n-propanol $60 \%(\mathrm{vol} / \mathrm{vol})$ proved to be more efficacious than isopropanol $70 \%$ ( $\mathrm{vol} / \mathrm{vol}$ ) when applied for a similar duration. ${ }^{8}$ In a recent study, ${ }^{4}$ we found that increasing the volume percentage concentrations of both alcohols by approximately $5 \%$ (by changing to weight percentage concentrations), together with a prolonged application time of 5 minutes, rendered the immediate effect of the 2 modified WHO formulations noninferior to the reference, thus conforming to the efficacy requirement of the norm. Unfortunately, this was not the case with the 3-hour effect, which remained inferior to the reference. This surprising result raised the suspicion that glycerol may be the reason for this phenomenon, at least in the given concentration of $1.45 \%$.

Glycerol is often added to hand hygiene preparations as a humectant or emollient to increase the acceptability, because frequent use of pure alcohol can cause dry skin. A positive effect of glycerol in alcohol-based hand rubs on skin condition and user acceptability has been shown by some authors. ${ }^{12,13}$ Kampf et al ${ }^{13}$ found that the addition of a mixture of emollients containing $0.81 \%$ (wt/wt) glycerol to a propanol-based hand rub resulted in significantly less dryness and erythema after frequent application. However, the influence of glycerol on the bactericidal efficacy of the alcoholbased hand rubs tested here has, to the best of our knowledge, not been investigated before. The original WHO-recommended formulations contain $1.45 \% \mathrm{vol} / \mathrm{vol}$ glycerol, which is a high concentration when compared with commercially available preparations. We suspected that this may have reduced the bactericidal efficacy that we $e^{4}$ and other authors ${ }^{3}$ have observed.

Indeed, as shown by the results of our study, reducing the glycerol concentration in the formulations or completely omitting glycerol increased the bactericidal efficacy, especially that of the isopropanol-based formulation, such that it conformed to the EN 12791 standard. The immediate effect of the isopropanol formulation without glycerol was even significantly stronger than that of the reference, which suggests sufficient efficacy when the application duration is shorter than 5 minutes. The 3-hour effect of both formulations with reduced glycerol content $(0.725 \%)$, compared with the original WHO-recommended formulations, was successfully rendered noninferior to the reference; the efficacy of the glycerolfree preparations. was even significantly stronger than that of the reference. Thus, it appears that the glycerol concentration in formulations for hand hygiene is critical. If it is too low or absent, frequent use may lead to skin dryness, but if it is too high, hands may feel sticky, and, more importantly, the hand rub bactericidal performance may be unfavorably influenced. The cause for this latter phenomenon is still unknown.

In conclusion, as shown by recent investigations, ${ }^{3,4}$ the original WHO-recommended formulations do not comply with the efficacy requirements of EN 12791. It may seem debatable that, in this official European norm, n-propanol, which is the most efficacious of the alcohols used in hand hygiene, is included as a reference, because it is only sparsely used in clinical practice worldwide, and there is no evidence that its efficacy as a reference finds a clinical correlate. ${ }^{1}$ However, it is to be considered that it is used only as a laboratory standard and serves as the yardstick for high-level bactericidal efficacy of surgical hand preparations.

Improving the immediate bactericidal efficacy of the original formulations is possible by increasing the alcohol concentrations by approximately $5 \%$ and prolonging the duration of application to 5 minutes. ${ }^{3}$ It is of note that an increase in the alcohol concentration has also been shown to be beneficial for the modified WHO formulations in their application as 
TABLE 2. Comparison of the 3-Hour Effects of a 5-Minute Application of Modified World Health Organization Antiseptic Hand Rub Formulations with 3-Minute Application of Reference Surgical Hand Antiseptic Treatment according to European Norm 12791

\begin{tabular}{|c|c|c|c|c|c|c|}
\hline \multirow[b]{2}{*}{ Formulation } & \multirow[b]{2}{*}{$\begin{array}{l}\text { Glycerol } \\
\text { added }\end{array}$} & \multicolumn{3}{|c|}{$\begin{array}{l}\text { Skin bacteria, cfu/mL } \\
(n=25)\end{array}$} & \multirow[b]{2}{*}{$\begin{array}{c}\text { Wilcoxon } \\
\text { matched-pairs test }{ }^{a}\end{array}$} & \multirow[b]{2}{*}{$\begin{array}{l}\text { Hodges-Lehmann upper } \\
97.5 \% \text { confidence limit, } \\
\log _{10}{ }^{b}\end{array}$} \\
\hline & & $\begin{array}{c}\text { Mean } \log _{10} \\
\text { prevalue }( \pm S D)\end{array}$ & $\begin{array}{c}\text { Mean } \log _{10} \\
\text { reduction }( \pm S D)\end{array}$ & $\begin{array}{c}\text { Mean } \log _{10} \\
\text { difference }( \pm S D), \\
\Delta, \text { to reference }\end{array}$ & & \\
\hline $\mathrm{EtOH} \mathrm{G+}$ & Yes & $4.67 \pm 0.53$ & $1.58 \pm 0.99$ & $-0.02 \pm 1.19$ & 11 & 0.48 n.i. \\
\hline $\mathrm{EtOH} \mathrm{G-}$ & No & $4.55 \pm 0.53$ & $2.04 \pm 1.15$ & $-0.48 \pm 1.02$ & $7^{\mathrm{c}}$ & -0.10 n.i. \\
\hline Iso $\mathrm{G}-$ & No & $4.54 \pm 0.55$ & $2.04 \pm 0.99$ & $-0.48 \pm 0.99$ & $8^{c}$ & -0.06 n.i. \\
\hline Reference & No & $4.41 \pm 0.81$ & $1.55 \pm 1.06$ & $\ldots$ & $\ldots$ & $\ldots$ \\
\hline
\end{tabular}

hygienic hand rubs, because their bactericidal efficacy has been proven to conform to another European standard for testing the bactericidal efficacy of hygienic hand rubs (EN 1500) when applied even for only 30 seconds, rather than 60 seconds, which is the duration of application necessary to comply with the original WHO-recommended formulations. ${ }^{14}$ This shortening of the necessary exposure time may help medical personnel to comply with hand hygiene. ${ }^{15}$

Despite their improved immediate effect in preoperative hand preparation, the modified formulations failed to conform to EN 12791 with respect to their 3-hour efficacy. ${ }^{4}$ Our findings indicate that it is probable that the high glycerol content in the formulations was responsible for this phenomenon.

With regard to the limitations of our study, it should be indicated that our results have not yet been confirmed by other laboratories and that interlaboratory variation is possible. Furthermore, the study was conducted only with the aim of harmonizing the bactericidal efficacy of WHO-recommended formulations with the requirements of EN 12791, although there is no evidence to date that better test performance is associated with better clinical outcome.

Nevertheless, we feel that changing the original ethanolbased formulation WHO I to $80 \%$ (wt/wt) ethanol, $0.125 \%$ (vol/vol) hydrogen peroxide, and $0.725 \%$ (vol/vol) glycerol should be considered. Likewise, we suggest that the original isopropanol-based WHO II formulation be recomposed to contain $75 \%$ (wt $/ \mathrm{wt}$ ) isopropanol, $0.125 \%$ (vol $/ \mathrm{vol}$ ) hydrogen peroxide, and $0.725 \%$ ( $\mathrm{vol} / \mathrm{vol}$ ) glycerol.

Although both new formulations were successfully tested for 5-minute application, one may speculate from our results that they could meet the efficacy requirements of the norm even with a 3-minute duration of application, which is more convenient and, at present, the most common duration of application for preoperative hand rubs in Europe. Additional studies of the influence of glycerol on the efficacy of pure ethanol, isopropanol, and n-propanol are being conducted at this time. It is of importance to note that information on the dermal tolerability and healthcare workers' acceptance of the modified formulations proposed here is not yet available. Adoption of the modified formulations for regular use in healthcare settings should be based on field testing, which is presently in progress in collaboration with the WHO. However, as long as there are no additional results available from studies dealing with this issue, formulations that do not contain glycerol should be regarded as experimental.

\section{ACKNOWLEDGMENTS}

Potential conflicts of interest. All authors report no conflicts of interest relevant to this article. All authors submitted the ICMJE Form for Disclosure of Potential Conflicts of Interest, and the conflicts that the editors consider relevant to this article are disclosed here.

Address correspondence to Miranda Suchomel, $\mathrm{PhD}$, Institute of Hygiene and Applied Immunology, Medical University of Vienna, Kinderspitalgasse 15, 1090 Vienna, Austria (miranda.suchomel@meduniwien.ac.at).

\section{REFERENCES}

1. World Health Organization (WHO). WHO Guidelines on Hand Hygiene in Health Care. Geneva: WHO, 2009.

2. European Norm (EN) 12791. Chemical disinfectants and antiseptics. Surgical hand disinfection: test method and requirement (phase 2/step 2). Brussels: Comité Européen de Normalisation, 2005.

3. Kampf G, Ostermeyer C. World Health Organization-recommended hand-rub formulations do not meet European efficacy requirements for surgical hand disinfection in five minutes. I Hosp Infect 2011;78(2):123-127.

4. Suchomel M, Kundi M, Allegranzi B, Pittet D, Rotter M. Testing of the World Health Organization-recommended formulations 
for surgical hand preparation and proposals for increased efficacy. J Hosp Infect 2011;79:115-118.

5. Lehmann EL. Nonparametrics: statistical methods based on ranks. San Francisco: Holden-Day, 1975.

6. Rotter ML. Hand washing and hand disinfection. In: Mayhall CG, ed. Hospital Epidemiology and Infection Control. 4th edition. Philadelphia: Wolters Kluwer, Lippincott, Williams and Wilkins, 2011:1365-1383.

7. Suchomel M, Gnant G, Weinlich M, Rotter M. Surgical hand disinfection using alcohol: the effects of alcohol type, mode and duration of application. J Hosp Infect 2009;71:228-233.

8. Suchomel M, Koller W, Kundi M, Rotter M. Surgical hand rub: Influence of duration of application on the immediate and 3hour effects of n-propanol and isopropanol. Am J Infect Control 2009;37:289-293.

9. Suchomel M, Rotter M. Ethanol in surgical hand rubs: concentration and duration of application for achieving European Norm EN 12791. J Hosp Infect 2011;77:263-266.

10. Kampf G, Ostermeyer C. Efficacy of two ethanol-based hand rubs for surgical hand disinfection: a controlled trial according to prEN 12791. BMC Inf Dis 2005;5:17.

11. Rotter ML, Kundi M, Suchomel M, et al. Reproducibility and workability of the European test standard EN 12791 regarding the effectiveness of surgical hand antiseptics: a randomized, multicenter trial. Infect Cont Hosp Epidemiol 2006;27:935-939.

12. Houben E, de Paepe K, Rogiers V. Skin condition associated with intensive use of alcoholic gels for hand disinfection: a combination of biophysical and sensorial data. Contact Dermatitis 2006;54:261-267.

13. Kampf G, Wigger-Alberti W, Schoder V, Wilhelm KP. Emollients in a propanol-based hand rub can significantly decrease irritant contact dermatitis. Contact Dermatitis 2005;53(6):344-349.

14. European Norm 1500. Chemical Disinfectants and Antiseptics: $H y$ gienic Hand Disinfection-Test Method and Requirement (Phase 2/ Step 2). Brussels: Comité Européen de Normalisation, 1997.

15. Suchomel M, Kundi M, Pittet D, Weinlich M, Rotter ML. Testing of the World Health Organization recommended formulations in their application as hygienic hand rubs and proposals for increased efficacy. Am J Infect Control 2012;40(4):328-331. 